\title{
Safety and efficacy of digital single-operator pancreatoscopy for obstructing pancreatic ductal stones $\square$
}

\section{다 (1) $\odot$}

Authors

Olaya I. Brewer Gutierrez¹, Isaac Raijman², Raj J. Shah ${ }^{3}$, B. Joseph Elmunzer ${ }^{4}$, George J.M. Webster ${ }^{5}$, Douglas Pleskow $^{6}$, Stuart Sherman ${ }^{7}$, Richard P. Sturgess ${ }^{8}$, Divyesh V. Sejpal ${ }^{9}$, Christopher Ko ${ }^{10}$, Attilio Maurano ${ }^{11}$, Douglas G. Adler $^{12}$, Daniel K. Mullady ${ }^{13}$, Daniel S. Strand ${ }^{14}$, Christopher J. DiMaio ${ }^{15}$, Cyrus Piraka ${ }^{16}$, Reem Sharahia ${ }^{17}$, Mohamad H. Dbouk ${ }^{1}$, Samuel Han ${ }^{3}$, Clayton M. Spiceland ${ }^{4}$, Noor L.H. Bekkali ${ }^{5}$, Moamen Gabr ${ }^{6}$, Benjamin Bick ${ }^{7}$, Laura K. Dwyer ${ }^{8}$, Dennis Han ${ }^{9}$, James Buxbaum ${ }^{10}$, Claudio Zulli ${ }^{11}$, Natalie Cosgrove ${ }^{13}$, Andrew Y. Wang ${ }^{14}$, David Carr-Locke ${ }^{17}$, Tossapol Kerdsirichairat ${ }^{1}$, Hanaa Dakour Aridi ${ }^{1}$, Robert Moran ${ }^{1}$, Shawn Shah ${ }^{17}$, Juliana Yang ${ }^{1}$, Omid Sanaei ${ }^{1}$, Nasim Parsa ${ }^{1}$, Vivek Kumbhari ${ }^{1}$, Vikesh K. Singh ${ }^{1}$, Mouen A. Khashab ${ }^{1}$

Institutions

1 Division of Gastroenterology and Hepatology, Johns Hopkins Hospital, Maryland United States

2 Greater Houston Gastroenterology, Texas, United States

3 Division of Gastroenterology and Hepatology University of Colorado-Denver Medical School, Colorado, United States

4 Division of Gastroenterology and Hepatology. Medical University of South Carolina, Charleston, South Carolina, United States

5 University College London Hospitals, London, UK

6 Division of Gastroenterology and Hepatology Beth Israel Deaconess Medical Center, Harvard Medical School, Boston, Massachusetts, United States

7 Division of Gastroenterology and Hepatology Indiana University Medical Center, Indiana, United States

8 Division of Gastroenterology and Hepatology, Aintree University Hospital, Liverpool, United Kingdom

9 Division of Gastroenterology and Hepatology HofstraNorthwell School of Medicine, New York, United States

10 Division of Gastroenterology and Hepatology University of Southern California Keck School of Medicine, California, United States

11 University Hospital of Salerno, G. Fucito Center, Mercato San Severino (SA), Italy

12 Division of Gastroenterology and Hepatology University of Utah School of Medicine, Utah, United States

13 Division of Gastroenterology and Hepatology, Washington University in St. Louis School of Medicine, St. Louis, Missouri, United States

14 Division of Gastroenterology and Hepatology University of Virginia Health System, Virginia, United States

15 Division of Gastroenterology Icahn School of Medicine at Mount Sinai, One Gustave L. Levy Place, New York, United States
16 Henry Ford Hospital, Detroit, Michigan, United States

17 Division of Gastroenterology and Hepatology Weil Cornell, New York, New York, United States

Bibliography

DOI https://doi.org/10.1055/a-0889-7743 |

Endoscopy International Open 2019; 07: E896-E903

(c) Georg Thieme Verlag KG Stuttgart · New York

eISSN 2196-9736

Corresponding author

Mouen A. Khashab, MD, Johns Hopkins Hospital, 1800

Orleans Street, Sheikh Zayed Tower, Baltimore, MD 21287

Fax: +1-410-502-7010

mkhasha1@jhmi.edu

\section{ABSTRACT}

Background and study aims The role of the digital single-operator pancreatoscopy (D-SOP) with electrohydraulic $(\mathrm{EHL})$ or laser lithotripsy (LL) in treating pancreatic ductal stones is unclear. We investigated the safety and efficacy of D-SOP with EHL or LL in patients with obstructing pancreatic duct stones.

Patients and methods Retrospective analysis of 109 patients who underwent D-SOP for pancreatic stones at 17 tertiary centers in the United States and Europe from February 2015 to September 2017. Logistic regression was performed to identify factors associated with the need for more than one D-SOP with EHL/LL.

Results Most patients were males (70.6\%), mean age 54.7 years. Fifty-nine (54.1\%) underwent EHL and 50 (45.9\%) underwent LL. Mean procedure time was longer in the EHL group (74.4 min vs $53.8 \mathrm{~min} ; P<0.001)$. Ducts were completely cleared (technical success) in $89.9 \%$ of patients ( $94.1 \%$ in $\mathrm{EHL}$ vs $100 \%$ in LL; $P=0.243$ ), achieved in a single session in $73.5 \%$ of patients $(77.1 \%$ by EHL and $70 \%$ by LL; $P=0.5)$.D-SOP failed in 11 patients (10.1\%); 6 patients were 
treated with extracorporeal shockwave lithotripsy (ESWL), 1 with surgery, 1 with combined treatment (ESWL +D-SOP $E H L)$ and 3 with other. Fourteen adverse events occurred in 11 patients $(10.1 \%)$. Patients with more than three ductal stones were more likely to have technical failure compared to those with less than three stones $(17 \%$ vs. $4.8 \%$; $P=$
0.04). Having more than three stones was independently associated with the need for more than one D-SOC EHL/LL session (OR 2.94, $95 \% \mathrm{Cl} 1.13-7.65$ ).

Conclusion D-SOP with EHL or LL is effective and safe in patients with pancreatic ductal stones.

\section{Introduction}

Chronic pancreatitis (CP) is associated with pancreatic duct stones in $50 \%$ to $90 \%$ of patients [1]. These stones may occur in the main pancreatic duct (MPD) or side branches (SB), and contribute to increased intraductal and parenchymal pressure [1 - 3]. The composition of pancreatic stones may include an inner nidus of nickel, iron, and chromium surrounded by successive layers of calcium carbonate. These constituents contribute to pancreatic stones being harder than bile duct stones, making endoscopic treatment often challenging, especially when combined with ductal strictures and angulations [1,3].

The goal of therapy in CP is symptom control and pain relief, with the aim of decompressing an obstructed main pancreatic duct and/or removal of obstructing stones. Historically, treatment has been endoscopic or surgical, with endoscopic treatment consisting of ERCP with pancreatic sphincterotomy/endoscopic papillary balloon dilation (EPBD), stone extraction using a retrieval basket and/or retrieval balloon and stent placement. Surgical treatment usually involves resection and drainage. Prior studies have shown that both treatments are effective although surgery is longer lasting [2,3]. Given the challenges in endoscopic treatment, Extracorporeal shockwave lithotripsy (ESWL) has been considered lately as the cornerstone treatment modality in symptomatic CP. Multiple studies have shown MPD clearance after ESWL, alone or in combination with ERCP, of $59 \%$ to $80 \%$ and long-term pain relief of $60 \%$ to $90 \%[1,3,4]$.

Per-oral pancreatoscopy (POP) enables direct visualization of the pancreatic duct. Although it has been available since the 1990s, it was not popular given technical difficulties, the requirement for two operators, and poor imaging quality. With the introduction of the single-operator cholangiopancreatoscopy system (SOCP) (SpyGlass; Boston Scientific, Natick, Massachusetts, United States) in 2007, the requirement for two operators was solved $[5,6]$. Nevertheless, it was underutilized due to suboptimal fiber optic imaging [7]. The digital version of SOC (D-SOCP) (SpyGlass DS; Boston Scientific), available since February 2015 , significantly improved image quality compared to the prior system, possibly increasing its diagnostic and therapeutic capabilities $[5,6]$. Electrohydraulic lithotripsy (EHL) or laser lithotripsy (LL) can be used during SOCP to achieve stone fragmentation, enabling complete ductal clearance during ERCP in a safe and efficient manner $[1,6,8,9]$. Our group previously reported the largest cohort of patients with difficult bile duct stones treated with D-SOC with EHL/LL with $97 \%$ efficacy in clearing the bile duct [6]. Presently, POP with EHL/LL is used mainly as a rescue therapy after failure of ESWL.
A few studies with small sample sizes or using non-digital pancreatoscopes have assessed the performance of EHL or LL in treatment of pancreatic stones, with a reported efficacy of $40 \%$ to $100 \%$ [1, $10-12]$. The new digital version of the SOP with improved image quality possibly aids stone fragmentation using the available lithotripsy devices. The primary aim of this study was to assess technical success, defined as pancreatic duct clearance, in patients with chronic pancreatitis and MPD stones. Secondary aims were to assess the safety of D-SOP with $\mathrm{EHL}$ or $\mathrm{LL}$ and to compare the effectiveness of EHL vs. $\mathrm{LL}$ in the largest cohort of patients with obstructing pancreatic stones to date.

\section{Patients and methods}

This was a retrospective, international, multicenter study including 17 tertiary centers (14 from the United States, 3 from Europe). Institutional Review Board approval was obtained at all institutions. Medical records were assessed to identify all consecutive adult patients (>18 years) who underwent D-SOP using either EHL or LL for management of obstructing pancreatic duct stones from February 2015 to September 2017.

The study included patients with symptomatic pancreatic stones, most of whom had prior attempts at stone clearance using standard ERCP techniques and/or ESWL. Intraductal stones were diagnosed by abdominal cross-sectional imaging or prior ERCP. Procedures done with the older version of SOP or other types of cholangiopancreatoscopes were excluded, as were all cases of D-SOP without EHL/LL. All procedures were performed under general anesthesia or propofol deep sedation. Procedure time was defined as the time between duodenoscope scope-in and scope-out. Patients were admitted to hospital for a planned 23-hour observation period after the index D-SOP. Nevertheless, when clinically appropriate, patients were discharged after a 1- to 2-hour period of observation. When the index D-SOP EHL/LL yielded partial or failed stone clearance, repeated D-SOP was attempted until complete clearance was achieved. The endoscopist determined stone clearance based on final pancreatogram or D-SOP at the time of the procedure.

The D-SOP (Spyglass DS, Boston Scientific) is a single-use 10.8 French (F) scope with four-way tip maneuverability, dedicated irrigation and aspiration channels, and one 1.2-mm working channel. It has enhanced visualization with a digital sensor $[4,5,7]$.

Several lasers have been developed. Laser light at a particular wavelength is focused on the surface of the stone to induce 
wave-mediated fragmentation [1]. In this study, the VersaPulse P20, Slim line 365- $\mu$ m fiber Holmium laser (Ho:YAG) (Lumenis Inc., San Jose, California, United States) was used. The power setting was maximum $20 \mathrm{~W}$ (starting at $1 \mathrm{~J}$ and $10 \mathrm{~Hz}$ then rising to $2.5 \mathrm{~J} \times 8 \mathrm{~Hz}$ ), in bursts of 5 seconds. Stone fragments were retrieved by standard techniques ( $\vee$ Fig.1) ( $\vee$ Supplemental Video 1).

$\mathrm{EHL}$ is a bipolar $1.9 \mathrm{~F}$ probe that discharges sparks with the aid of a charge generator (AUTOLITH, Northgate Technologies Inc., Illinois, United States) in an aqueous medium (normal saline). The probe is positioned 1 to $2 \mathrm{~mm}$ from the stone. The spark produced under the saline medium generates high-frequency hydraulic pressure waves, absorbed by nearby stones resulting in their fragmentation. Shock waves are delivered in brief pulses, ranging from a single discharge to continuous firing $[1,5]$. The stone fragments were retrieved by conventional ERCP techniques. The power settings in the generator ranged between low/medium/high (50-100\%) and delivered over 1to 2-second bursts ( $\triangleright$ Fig. 2) ( $\$$ Supplemental Video 2 ).

The type of lithotripsy device used in the current study was chosen solely based on endoscopist preference and the center's availability.

\section{Outcomes and definitions}

The primary study outcome was rate of technical success, defined as complete pancreatic duct clearance. Safety of D-SOP using either EHL or LL was defined by the rate and severity of adverse events (AEs) as graded per the ASGE lexicon (mild, moderate, severe, fatal) [13]. Other outcomes included clinical success (defined by resolution or improvement of symptoms), number of D-SOP with EHL/LL sessions needed to clear the MPD, need for other therapies (ESWL or surgery), incomplete stone removal/stone recurrence and procedure time. Comparison of outcomes between EHL vs. LL was also assessed.

\section{Statistical analysis}

Descriptive statistics was presented as frequencies (\%) for categorical variables and mean \pm standard deviation (SD) or median (interquartile range [IQR]) for continuous variables. Chi-square test and Fisher's exact test were used to compare categorical data while Student's $t$-test and Wilcoxon rank-sum test were used for continuous data, as appropriate. A $P$ value $\leq 0.05$ was considered statistically significant. Logistic regression analysis was performed to identify factors associated with the need for more than one session of D-SOP with EHL/LL. Statistical analysis was performed using Stata software (Stata version 14.1, College Station, Texas, United States).

\section{Results}

A total of 109 patients ( $71 \%$ male, mean age $54.7 \pm 15.0$ years) underwent D-SOP with EHL or LL for pancreatic ductal stones. Most common presenting symptoms were abdominal pain (96.3\%) and/or weight loss (51.4\%). Diabetes was present in 48.6\%; $74.3 \%$ were on pancreatic enzymes and $74.3 \%$ on opioids. Most patients (88.1\%) had prior ERCP attempts and a minority (11\%) prior ESWL session(s) with failed ductal clear-

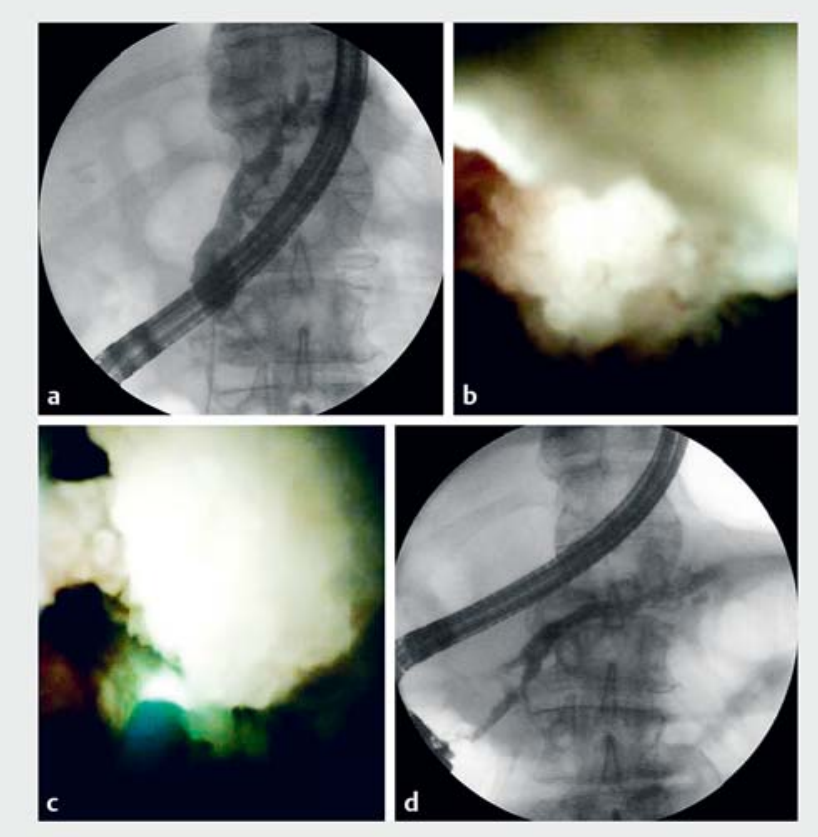

- Fig. 1 63-year-old female with history of alcohol induced chronic pancreatitis. Patient has recurrent post prandial abdominal pain. CT scan showed dilated MPD with stones. a ERCP showing a dilated, tortuous MPD with multiple filling defects at the genu/ body. $\mathbf{b}$ Intraductal pancreatic stone seen on pancreatoscopy. c Ho:YAG lasers' probe green light on the surface of the stone. d Final ERCP pancreatogram showing decompressed MPD without filling defects.

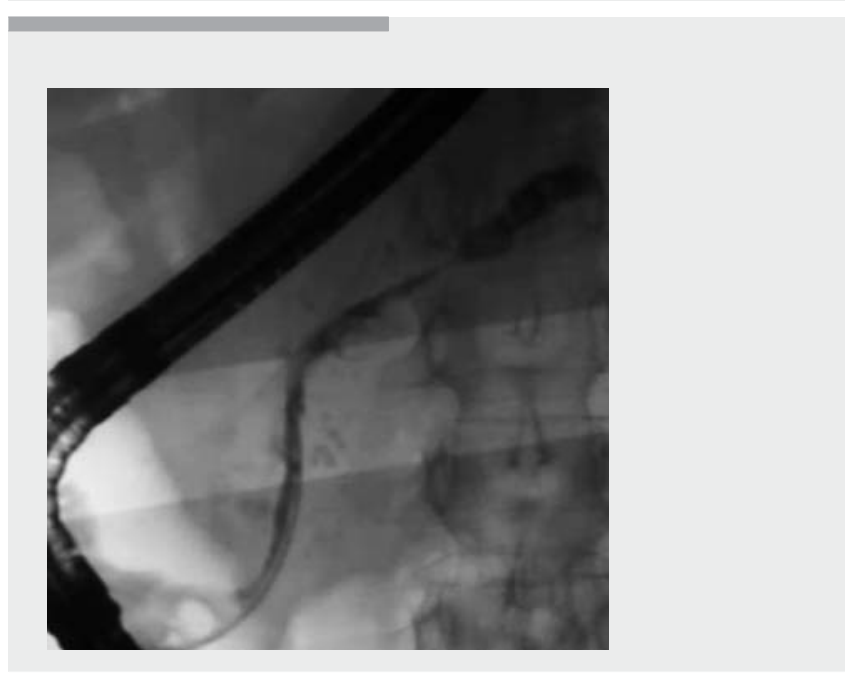

Video 1 DSOP with LL for treatment of MPD stone.

ance. The most frequent prior intervention was stone extraction using a retrieval balloon (73.4\%). Seventy-four patients (67.9\%) had a plastic pancreatic stent placed during a prior failed ERCP. Mean MPD diameter by prior imaging was $9.2 \pm$ $3.5 \mathrm{~mm}$ ( Table 1 ).

During the index D-SOP, all patients had successful MPD cannulation with the D-SOP. Image quality of stone visualization 

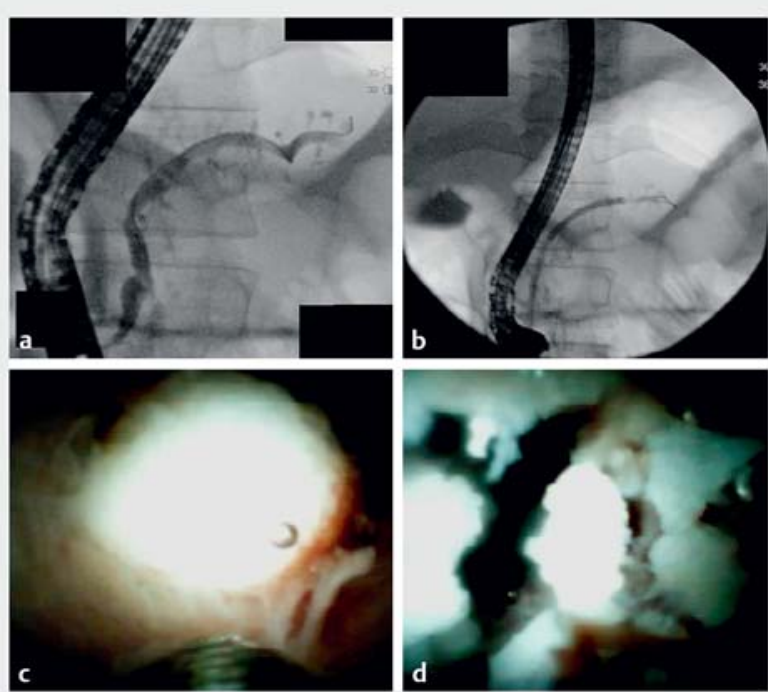

Fig. 2 33-year-old male with recurrent acute on chronic pancreatitis secondary to heterozygous SPINK1 N34S mutation. On CT scan, a stone located in the MPD at the level of the pancreatic body with upstream ductal dilation was noted. a ERCP showed a mildly dilated and tortuous MPD with side branches widening. The distal body/tail is not visualized despite high quality pancreatogram and attempts to pass the wire distally were unsuccessful. b The pancreatoscope was advanced over the wire to the area that was not well visualized. c A round stone was seen on pancreatoscopy. EHL probe advanced. d Fragments of the stone post lithotripsy.

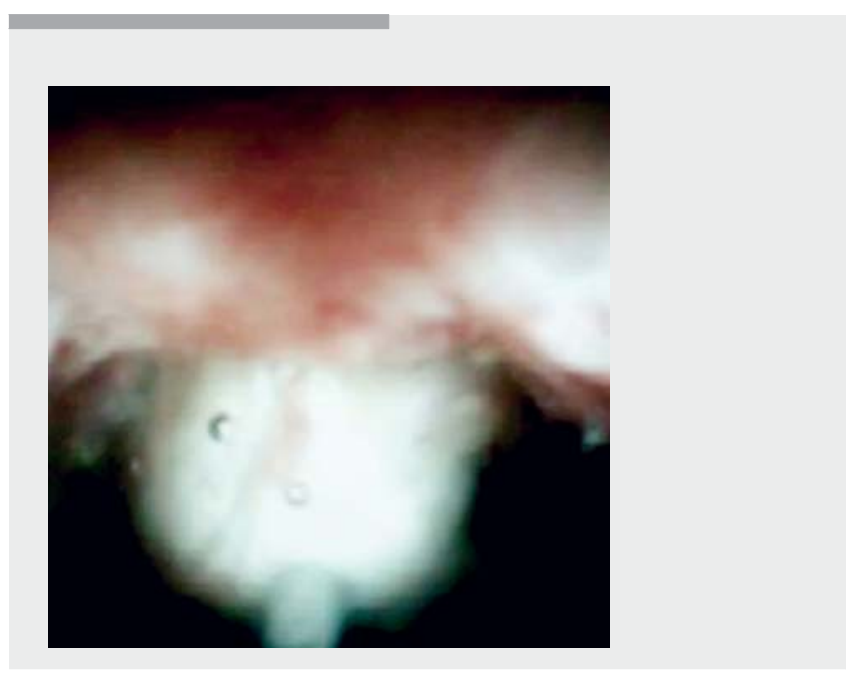

Video 2 DSOP with EHL for treatment of MPD stone.

was reported as excellent in $67.9 \%$ of cases and good in $30.3 \%$. In two cases (1.8\%), visualization was reported as poor, with stones located in the MPD and side branches. The majority of stones were located in the head of pancreas (HOP) (49.5\%), followed by the neck/genu (21.1\%), body (13.8\%), tail (5.5\%), and $10.1 \%$ were multifocal. Almost half of the cohort had more than three stones (43.2\%). Most stones (56.9\%) measured between
1 and $9 \mathrm{~mm}$ and $61.5 \%$ were impacted. Most patients (78\%) had a downstream MPD stricture, the majority (87.1\%) of whom required stricture dilation to perform lithotripsy. After $\mathrm{EHL}$ or $\mathrm{LL}$, the most common device used for stone extraction was the extraction balloon (83.5\%). Stents were placed after $\mathrm{D}-\mathrm{SOP}$ in $98.2 \%$ of patients. Mean procedure time (available in $78 \%$ of patients) was $62.8 \pm 23$ minutes ( $\triangleright$ Table 1 ).

\section{Outcomes}

Complete ductal clearance using EHL/LL (technical success) was achieved in $89.9 \%$ of patients, and in $73.5 \%$ was accomplished in a single session ( $\triangleright$ Table 2 ). Overall, success rates were similar among tertiary centers participating in this study. Half of patients needing more than one session had more than three stones (15/26), and stones were larger than $10 \mathrm{~mm}$ in $53.8 \%$ (14/26). Moreover, $81 \%$ of these patients $(21 / 26)$ had a downstream stricture (i.e. between the ampulla and the stone). Eleven patients $(10.1 \%)$ failed DSOP and were treated with surgery $(n=1)$, ESWL alone $(n=6)$, combined treatment with ESWL/DSOP EHL $(n=1)$ and other $(n=3)($ ERCP/balloon sweep and stenting $n=2$, awaiting treatment $n=1$ ). Reported reasons for technical failure were: failed stone fragmentation $(n=3)$, failure to advance $\mathrm{EHL}$ probe to the site of the stone $(n=3)$, failure to advance the D-SOP due to angulation/tight stricture $(n=3)$ and unspecified reason $(n=2)$. Of the 11 patients with technical failure, three were attributed to failure of stone fragmentation by EHL. There were no failures of fragmentation in the LL group. Patients with technical failure had more than three stones (8/11), impacted stones (7/11) and a downstream stricture (6/11). Of the 11 patients with technical failure, one required surgery (Puestow procedure). This patient had more than three ductal stones, impacted stones, and a downstream stricture. Clinical success was achieved in $88.4 \%$ of the patients.

Fourteen adverse events (AEs) occurred in 11 patients (10.1\%) including five pancreatitis, one MPD perforation, two bleeding, three fever and three abdominal pain without pancreatitis. These were rated as mild $(n=12,85.7 \%)$ and moderate $(n=2,14.3 \%)$, per ASGE lexicon. Ten patients (10/11) with AEs were admitted to the hospital. Most were treated conservatively with intravenous fluids, pain medication, and antibiotics, while five (5/11) were treated endoscopically with repeated ERCP and stent placement/exchange.

\section{Predictors of outcomes}

Patients with more than three stones were more likely to have technical failure compared to those with less than three stones (17\% vs. $4.8 \%, P=0.04$ ). Similarly, patients with more than three stones were more likely to need more than one D-SOP $\mathrm{EHL} / \mathrm{LL}$ session ( $38.5 \%$ vs. $18.6 \%, P=0.03$ ). Multivariable analysis was not possible for technical failure due to the small number of events $(n=11)$. On the other hand, the only factor associated with the need for more than one session of D-SOP EHL/LL was having more than three ductal stones (OR 2.94, $95 \% \mathrm{Cl}$ $1.13-7.65$ ), independently to the type of procedure (EHL vs. $\mathrm{LL}$ ) and the age of the patient ( $\triangleright$ Table 3 ). 
- Table 1 Patient baseline characteristics.

\begin{tabular}{|c|c|c|c|c|}
\hline & Total $(\mathrm{N}=109)$ & $\mathrm{EHL}(\mathrm{N}=59)$ & LL $(N=50)$ & $P$ value \\
\hline Female sex; n (\%) & $32(29.4)$ & $23(39)$ & $9(18)$ & 0.02 \\
\hline Mean age (mean $\pm S D$ ) & $54.7 \pm 15$ & $56 \pm 16$ & $53 \pm 13$ & 0.3 \\
\hline \multicolumn{5}{|l|}{ Symptoms } \\
\hline Abdominal pain; n (\%) & $105(96.3)$ & $56(94.9)$ & $49(98)$ & 0.62 \\
\hline Weight loss; n (\%) & $56(51.4)$ & $16(27.1)$ & $40(80)$ & $<0.001$ \\
\hline On pancreatic enzymes; $\mathbf{n}(\%)$ & $81(74.3)$ & $37(62.7)$ & $44(88)$ & 0.003 \\
\hline Opioid use; n (\%) & $81(74.3)$ & $39(66.1)$ & $42(84)$ & 0.05 \\
\hline Diabetes; n (\%) & $53(48.6)$ & $20(33.9)$ & $33(66)$ & 0.001 \\
\hline Exocrine pancreatic insufficiency; $\mathbf{n}(\%)$ & $46(42.2)$ & $15(25.4)$ & $31(62)$ & $<0.001$ \\
\hline Prior ESWL & $12(11)$ & $10(16.9)$ & $2(4)$ & 0.03 \\
\hline Patients from community (not center) & $68(62.4)$ & $26(44.1)$ & $42(84)$ & $<0.001$ \\
\hline Prior ERCP with failed stone extraction; $n$ (\%) & $96(88.1)$ & $46(78)$ & $50(100)$ & $<0.001$ \\
\hline \multicolumn{5}{|l|}{ Prior interventions for stone removal } \\
\hline Balloon extraction; $\mathrm{n}(\%)$ & $80(73.4)$ & $36(61)$ & $44(88)$ & 0.001 \\
\hline Retrieval basket; $\mathrm{n}(\%)$ & $13(11.9)$ & $8(13.6)$ & $5(10)$ & 0.57 \\
\hline $\mathrm{EHL} ; \mathrm{n}(\%)$ & $10(9.2)$ & $4(6.8)$ & $6(12)$ & 0.51 \\
\hline LL; n (\%) & $6(5.5)$ & $0(0)$ & $6(12)$ & 0.008 \\
\hline Prior surgery; n (\%) & $1(0.9)$ & $1(1.7)$ & $0(0.0)$ & 1 \\
\hline Indwelling pancreatic stent; $\mathbf{n}(\%)$ & $74(67.9)$ & $42(71.2)$ & $32(64)$ & 0.54 \\
\hline Main pancreatic duct size; (mean \pm SD) $(\mathrm{mm})$ & $9.2+3.5$ & $8.3+2.9$ & $10.1+3.9$ & 0.008 \\
\hline \multicolumn{4}{|l|}{ Stone location } & 0.16 \\
\hline Head; n (\%) & $54(49.5)$ & $35(59.3)$ & $19(38)$ & \\
\hline Neck; n (\%) & $23(21.1)$ & $10(16.9)$ & $13(26)$ & \\
\hline Body; n (\%) & $15(13.8)$ & $6(10.2)$ & $9(18)$ & \\
\hline Tail; n (\%) & $6(5.5)$ & $4(6.8)$ & $2(4)$ & \\
\hline Multifocal; n (\%) & $11(10.1)$ & $4(6.8)$ & $7(14)$ & \\
\hline \multicolumn{4}{|l|}{ Stone size (largest) } & 0.04 \\
\hline$<10 \mathrm{~mm} ; \mathrm{n}(\%)$ & $62(56.9)$ & $40(67.8)$ & $22(44)$ & \\
\hline $10-19$ mm; n (\%) & $32(29.4)$ & $13(22)$ & $19(38)$ & \\
\hline >20 mm; n (\%) & $15(13.8)$ & $6(10.2)$ & $9(18)$ & \\
\hline More than 3 stones in MPD; $\mathbf{n}$ (\%) & $47(43.1)$ & $30(50.8)$ & $17(34)$ & 0.077 \\
\hline Stone impaction; $\mathbf{n}(\%)$ & $67(61.5)$ & $26(44.1)$ & $41(82)$ & $<0.001$ \\
\hline Main pancreatic duct stricture; n (\%) & $85(78)$ & $39(66.1)$ & $46(92)$ & 0.001 \\
\hline \multicolumn{4}{|l|}{ Devices used for stone extraction } & 0.02 \\
\hline Extraction balloon & $91(83.5)$ & $44(74.6)$ & $47(94)$ & \\
\hline Retrieval basket & $5(4.6)$ & $5(8.5)$ & $0(0)$ & \\
\hline Other & $2(1.8)$ & $2(3.4)$ & $0(0)$ & \\
\hline Balloon + Basket & $7(6.4)$ & $4(6.8)$ & $3(6)$ & \\
\hline None & $4(3.7)$ & $4(6.8)$ & $0(0)$ & \\
\hline
\end{tabular}

EHL, electrohydraulic lithotripsy; LL, laser lithotripsy; SD, standard deviation; ERCP, endoscopic retrograde cholangiopancreatography; MPD, main pancreatic duct 
- Table 2 Procedure outcomes.

\begin{tabular}{|c|c|c|c|c|}
\hline & Total $(\mathrm{N}=109)$ & EHL $(N=59)$ & $\operatorname{LL}(N=50)$ & $P$ value \\
\hline Technical success (pancreatic duct clearance); n (\%) & $98(89.9)$ & $48 / 51^{1}(94.1)$ & $50(100)$ & 0.243 \\
\hline Clinical Success; $\mathbf{n}(\%)$, n= 95 & $84(88.4)$ & $40(88.9)$ & $44(88)$ & 1 \\
\hline \multicolumn{4}{|c|}{ Number of EHL/LL sessions to clear pancreatic duct; $n(\%)(n=98)$} & 0.5 \\
\hline 1 & $72(73.5)$ & $37(77.1)$ & $35(70)$ & \\
\hline $2-3$ & $23(23.5)$ & $9(18.8)$ & $14(28)$ & \\
\hline More than 3 & $3(3.1)$ & $2(4.2)$ & $1(2.0)$ & \\
\hline \multicolumn{4}{|c|}{ Number of additional ERCPs for dilation/removing stents; $n(\%)(n=90)$} & $<0.001$ \\
\hline None & $12(13.3)$ & $12(27.3)$ & $0(0.0)$ & \\
\hline 1 & $52(57.8)$ & $19(43.2)$ & $33(71.7)$ & \\
\hline $2-3$ & $17(18.9)$ & $8(18.2)$ & $9(19.6)$ & \\
\hline More than 3 & $9(10)$ & $5(11.4)$ & $4(8.7)$ & \\
\hline Stone recurrence; $n(\%)(n=89)$ & $9(10.1)$ & $6(14)$ & $3(6.5)$ & 0.3 \\
\hline \multicolumn{4}{|l|}{ Management of stone recurrence $(n=9)$} & 0.36 \\
\hline Balloon/basket; n (\%) & $4(44.4)$ & $3(50)$ & $1(33.3)$ & \\
\hline Cholangioscopy with EHL/LL; n (\%) & $1(11.1)$ & $1(16.7)$ & $0(0)$ & \\
\hline ESWL; n (\%) & $1(11.1)$ & $0(0)$ & $1(33.3)$ & \\
\hline Other; n (\%) & $1(11.1)$ & $0(0)$ & $1(33.3)$ & \\
\hline None; n (\%) & $2(22.2)$ & $2(33.3)$ & $0(0)$ & \\
\hline Procedure time $(\min )($ mean $\pm S D)(n=85)$ & $62.8+23$ & $74.4 \pm 25.5$ & $53.8 \pm 16.2$ & $<0.001$ \\
\hline Number of patients with adverse events; $n(\%)(n=11)$ & $11(10.1)$ & $5(8.5)$ & $6(12)$ & 0.54 \\
\hline Median follow up time; days median (IQR) & $210(68-387)$ & $157(63-353)$ & $291(141-410)$ & 0.005 \\
\hline Median time to recurrence; days $(I Q R)(n=9)$ & $105(85-471.5)$ & $91(76.25-205.5)$ & $448\left(105-{ }^{2}\right)$ & 0.5 \\
\hline \multicolumn{5}{|c|}{$\begin{array}{l}\text { EHL, electrohydraulic lithotripsy; LL, laser lithotripsy; ESWL, extracorporeal shockwave lithotripsy; SD, standard deviation. } \\
{ }^{1} \text { Eight technical failures were due to reasons not specific to the EHL procedure per se and thus were excluded from the comparison of EHL vs LL in technical success. } \\
{ }^{2} \text { There were only three recurrences. There is no interquartile range. }\end{array}$} \\
\hline
\end{tabular}

- Table 3 Factors associated with the need of more than one DSOP with $\mathrm{EHL} / \mathrm{LL}$.

\begin{tabular}{|c|c|c|}
\hline \multirow[t]{2}{*}{ Factors } & \multicolumn{2}{|c|}{$\begin{array}{l}\text { More than one D-SOP with EHL/LL } \\
(n=26)\end{array}$} \\
\hline & Odds Ratio (95\% CI) & $P$ value \\
\hline EHL vs. LL & $0.64(0.24-1.67)$ & 0.36 \\
\hline $\begin{array}{l}\text { More than } 3 \text { ductal } \\
\text { stones }\end{array}$ & $2.94(1.13-7.65)$ & 0.04 \\
\hline Age $>55$ years & $0.42(0.16-1.09)$ & 0.08 \\
\hline
\end{tabular}

EHL, electrohydraulic lithotripsy; LL, laser lithotripsy

\section{Incomplete stone removal/recurrent stones}

Median follow-up time was 210 days [IQR:68-387]. Patients with technical failure or those lost to follow-up were not included in this aspect of the analysis (20/109). After the index D-SOP $\mathrm{EHL} / \mathrm{LL}, 86.7 \%$ required at least an additional ERCP for stent removal/stent replacement and/or stricture dilation, and 13.4\% are waiting for a follow-up ERCP. Incomplete stone clearance/ recurrent stones was seen in nine of 89 patients $(10 \%)$ after a median follow-up time of 105 days [IQR:85-471.5]. Most of these patients had more than three stones and stone location was in the neck/genu, body, tail or multifocal (6/9).

Management of incomplete stone removal/ recurrent stones was performed with ERCP with extraction balloon ( $n=4,44.4 \%$ ), repeated D-SOC with EHL/LL ( $n=1,11.1 \%)$, ESWL ( $n=1,11.1 \%$ ), no treatment/expectant conduct $(n=2,22.2 \%)$ and in one patient $(11.1 \%$ ) treatment was not specified ( $\bullet$ Table 2 ). 


\section{EHL vs. LL}

Technical success was higher in the LL group (100\% vs. $94.1 \%$, $P=0.243$ ), although the difference was not significant. EHL procedures were significantly longer than those where LL was utilized $(74.4 \pm 25.5 \mathrm{~min}$. vs. $53.8 \pm 16.2 \mathrm{~min}, P<0.001)$. On the other hand, the number of patients with AEs were not significantly different between the two groups ( $8.5 \%$ vs $12 \%, P=$ $0.54)$. Moreover, in both groups, most patients needed one session to achieve MPD clearance $(70.0 \%$ vs. $77.1 \%, P=0.49)$ and clinical success was similar as well (88 vs $88.9 \%, P=1$ ) ( $\triangleright$ Table2).

\section{Discussion}

Endoscopic management of pancreatic ductal stones can be challenging, due to stone size, density, and location. Small and floating calculi less than $5 \mathrm{~mm}$ can be extracted by standard techniques of ERCP with pancreatic sphincterotomy followed by balloon trawl or basket. Stones greater than $5 \mathrm{~mm}$ are often impacted and difficult to extract using standard techniques [3]. Reported rates of stone clearance using the basket have been as low as $9 \%[3,14]$. Larger, impacted pancreatic stones require pre-extraction fragmentation using ESWL or other lithotripsy method, EPBD or surgical procedures. Factors that decrease the rate of endoscopic clearance include surgically altered anatomy, strictures, ductal angulation, and impacted stones [1]. ESWL alone or in combination with ERCP is reported to have an efficacy in clearing pancreatic stones of $59 \%$ to $80 \%$ [1]. Nevertheless, there are no definitive data on the number of sessions needed, and a subsequent ERCP is often performed after the last session to clear stone fragments, treat strictures, and place a stent, incurring additional costs. The rate of AEs of ESWL has been reported between $5.8 \%$ and $6.7 \%$ [1,2]. Complications include pain and ecchymosis at the site of shockwave, abdominal pain, and fever. Rare AEs include perirenal hematoma, biliary obstruction, splenic rupture, bowel perforation, liver trauma, and necrotizing pancreatitis [1,2]. Moreover, ESWL units are not typically within the endoscopy unit, requiring inter-departmental referrals, potentially delaying therapy.

D-SOC EHL/LL has been reported to be highly effective and safe in treating difficult bile duct stones [6] and previous small studies/case reports using POP in management of pancreatic stones have shown acceptable rates of success and safety [7, $10,12]$. The introduction of the D-SOC has improved the ease of SOC and markedly improved image quality. These have led to a greater utilization of EHL or LL in the ERCP armamentarium for management of difficult biliary and now pancreatic stones.

The largest study to date on SOP-LL in management of pancreatic stones was published by Atwell et al [12]; a retrospective multicenter study including 28 patients, with 79\% complete ductal clearance and $11 \%$ partial stone clearance rate. Stone clearance was achieved in one session in $61 \%$ of patients and AEs were noted in $29 \%$ of patients (post-ERCP pancreatitis and abdominal pain). A median of two stones sized $15 \mathrm{~mm}$ were identified in the head (32\%), neck (11\%), body (32\%), tail ( $4 \%)$, or multifocal $(21 \%)$; stone clearance was greater when stones were located in the head (92\%) compared to the tail (67\%). Main limitations of the study were small number of patients included and lack of information regarding reasons of prior ERCP or ESWL failures.

A systematic review performed by Beyna et al [1], assessed the efficacy and safety of POP-guided EHL and LL in pancreatic stones, including both SOP versions (DS and Legacy) and other types of pancreatoscopes, with a total of 87 patients. The rate of successful ductal clearance was $43 \%$ to $100 \%$ with $0 \%$ to $13.5 \%$ AEs. Limitations were the heterogeneity of the studies and the variation in the type of pancreatoscopes.

Our multicenter study is the largest to date on use of D-SOP with EHL or LL in management of pancreatic stones, showing high efficacy and safety. Ductal clearance was seen in $89.9 \%$ of patients, and in $73.5 \%$ was achieved in a single session. Clinical success was seen in $88.4 \%$ of patients. AEs were seen in $10.1 \%$ of patients, the majority of which were pancreatitis $(n=5)$, bleeding $(n=3)$ and abdominal pain $(n=3)$, and managed conservatively in the majority of cases. When comparing EHL to LL, most outcomes were similar except for procedure time, which was longer in the EHL group, with statistical significance (74.4 min vs. $53.8 \mathrm{~min}, P<0.001$ ). Pancreatic stones are often harder than bile duct stones (i.e Hounsfield index $>2000 \mathrm{HU}$ ). The advantage of LL over EHL in terms of rate of success and requiring less procedure time theoretically could be explained by the ability of LL to fragment denser stones $[10,12]$. Nevertheless, it is worth noting that LL technology is more expensive than EHL. Moreover, we need larger studies to validate our results. Regarding AEs, both techniques have similar AEs profiles, more frequent when compared to cholangioscopy and lithotripsy, mainly due to the higher risk of post ERCP pancreatitis, which is similar to risk of pancreatitis after standard pancreatic ERCP techniques (7-15\%). Independently of the chosen lithotripsy technique, it is important to avoid long periods of pancreatic duct exposure to high energy levels in a single session, given the risk of thermal injury to the ductal wall.

Limitations of this study are its retrospective nature, introducing possible selection bias. Although we collected information about prior procedures before D-SOP-EHL/LL, the choice of first-line treatment of pancreatic stones varies among centers and future randomized controlled trials comparing conventional ERCP techniques to ESWL and D-SOP EHL/LL are needed to clearly define the exact role and timing of this technique. Also, there was lack of information regarding the reasons for ERCP/ ESWL failures. Both groups differed in baseline characteristics which could affect the observed differences in outcomes. In addition, there were no objective measurements to assess pain or other symptoms before and after the procedure, making it difficult to accurately define the effect of D-SOP EHL/LL on symptom resolution. Even though our analysis showed that there are certain factors that could be associated with failure of D-SOP, the low number of patients with technical failure $(n=11)$ and the fact that there were no LL failures did not provide enough power to identify significant factors by multivariable analysis. Nevertheless, to our knowledge, this is the largest multicenter study on D-SOP with EHL/LL to date, involving 17 tertiary institutions. Our results may only be applicable to tertiary centers 
with high experience in chronic pancreatitis and D-SOP. Additionally, given the costs of these technologies, cost-effectiveness analysis comparing both techniques to ESWL and surgery is warranted.

\section{Conclusion}

In conclusion, D-SOP with either EHL or LL may represent an efficient, safe, and minimally invasive alternative to ESWL and surgical approaches in management of obstructing pancreatic ductal stones. When comparing both techniques, they have similar efficacy and safety profile.

\section{Competing interests}

Dr. Raijman is a consultant and speaker for Boston Scientific and Covidien and co-owner of EndoRx. Dr. Shah is consultant for Cook and for Boston Scientific. Dr. Webster is a consultant for Boston Scientific. Dr. Pleskow is a consultant for Boston Scientific, Olympus and Medtronic. Dr. Sherman is a consultant for Boston Scientific. Dr. Sturgess has received financial support from Boston Scientific to attend scientific meetings. Dr. Sejpal is a consultant for Boston Scientific, Olympus, and Ninepoint. Dr. Adler is a consultant for Boston Scientific. Dr. Mullady is a consultant for Boston Scientific and speaker for Abbvie. Dr. DiMaio is a consultant for Boston Scientific. Dr. Sharahia is a consultant for Boston Scientific and Apollo Endosurgery. Dr. Han has received $\mathrm{NIH}$ training grant $(\mathrm{NIH}$ T32DK007038). Dr. Bekkali has received a travel grant to attend UEGW 2016 from Boston Scientific. Dr. Wang has received research support from Cook Medical. Dr. Carr-Locke is a consultant for Boston Scientific and shares royalty from Steris and Telemed. Dr. Kumbhari is a consultant for Boston Scientific, ReShape Life Sciences, Apollo Endosurgery and Medtronic. Dr. Singh is a consultant for Abbvie, Akcea Therapeutics, and Ariel Precision Medicine. Dr. Khashab is a consultant for Boston Scientific, Olympus and Medtronic and is on the medical advisory board for Boston Scientific and Olympus.

\section{References}

[1] Beyna T, Neuhaus H, Gerges C. Endoscopic treatment of pancreatic duct stones under direct vision: Revolution or resignation? Systematic review Dig Endosc 2018; 30: 29-37

[2] Díte P, Ruzicka M, Zboril $\vee$ et al. A prospective, randomized trial comparing endoscopic and surgical therapy for chronic pancreatitis. Endoscopy 2003; 35: 553-558

[3] Tandan M, Talukdar R, Reddy DN. Management of pancreatic calculi: an update. Gut Liver 2016; 10: 873-880

[4] Dumonceau JM, Costamagna G, Tringali A et al. Treatment for painful calcified chronic pancreatitis: extracorporeal shock wave lithotripsy versus endoscopic treatment: a randomized controlled trial. Gut 2007; 56: $545-552$

[5] Komanduri S, Thosani N. ASGE Technology Committee. et al. Cholangiopancreatoscopy. Gastrointest Endosc 2016; 84: 209-221

[6] Brewer Gutierrez OI, Bekkali NLH, Raijman I et al. Efficacy and safety of digital single-operator cholangioscopy for difficult biliary stones. Clin Gastroenterol Hepatol 2018; 16: 918-926

[7] Navaneethan U, Hasan MK, Kommaraju K. Digital, single-operator cholangiopancreatoscopy in the diagnosis and management of pancreatobiliary disorders: a multicenter clinical experience. Gastrointest Endos 2016; 84: 649-655

[8] Shah RJ, Raijman I, Brauer B et al. Performance of a fully disposable, digital, single-operator cholangiopancreatoscope. Endoscopy 2017; 49: $651-658$

[9] Stefanidis G, Christodoulou C, Manolakopoulos S et al. Endoscopic extraction of large common bile duct stones: A review article. World J Gastrointest Endosc 2012; 4: 167-179

[10] Bekkali NL, Murray S, Johnson G] et al. Pancreatoscopy-directed electrohydraulic lithotripsy for pancreatic ductal stones in painful chronic pancreatitis using SpyGlass. Pancreas 2017; 46: 528 - 530

[11] Pereira P, Peixoto A, Andrade P et al. Peroral cholangiopancreatoscopy with the SpyGlass ${ }^{\circledR}$ system: what do we know 10 years later. J Gastrointestin Liver Dis 2017; 26: 165-170

[12] Attwell AR, Patel S, Kahaleh M et al. ERCP with per-oral pancreatoscopy-guided laser lithotripsy for calcific chronic pancreatitis: a multicenter U.S. experience. Gastrointest Endosc 2015; 82: 311 - 318

[13] Cotton PB, Eisen GM, Aabakken L et al. A lexicon for endoscopic adverse events: report of an ASGE workshop. Gastrointest Endosc 2010; 71: $446-454$

[14] Farnbacher MJ, Schoen C, Rabenstein T et al. Pancreatic duct stones in chronic pancreatitis: criteria for treatment intensity and success. Gastrointest Endosc 2002; 56: 501-506 